\title{
ons the \\ Measured Propagation Characteristics of Finite Ground Coplanar Waveguide on Silicon with a Thick Polyimide Interface Layer
}

\author{
George E. Ponchak, John Papapolymerou, Emmanouil M. Tentzeris
}

\begin{abstract}
Measured propagation characteristics of Finite Ground Coplanar (FGC) waveguide on silicon substrates with resistivities spanning 3 orders of magnitude (0.1 to $\mathbf{1 5 . 5}$ $\mathrm{Ohm} \mathrm{cm}$ ) and a $20 \mu \mathrm{m}$ thick polyimide interface layer is presented as a function of the FGC geometry. Results show that there is an optimum FGC geometry for minimum loss, and silicon with a resistivity of $0.1 \mathrm{Ohm} \mathrm{cm}$ has greater loss than substrates with higher and lower resistivity. Lastly, substrates with a resistivity of $10 \mathrm{Ohm} \mathrm{cm}$ or greater have acceptable loss.
\end{abstract}

Index Terms-silicon, coplanar waveguide (CPW), attenuation, interconnects

\section{INTRODUCTION}

Radio Frequency and Microwave Monolithic Integrated Circuits (RFICs and MMICs) fabricated on silicon substrates have obtained widespread use in personal communication, GPS, and other systems that are highly dependent on cost. While some of this market is due to reductions of CMOS gate width, the rest of it is due to the development of SiGe Heterojunction Bipolar Transistors (HBTs). Both of these technologies have pushed the frequency range of silicon RFICs to X-Band for commercial parts and Ka-Band and V-Band for research circuits. However, transmission lines and passive circuit components fabricated directly on standard, lowresistivity silicon wafers commonly used in commercial foundries have high loss, or low Q-factors [1]. Therefore, the transmission lines and passive components must be designed to shield the electric fields from the silicon as is done in thin film microstrip lines [2], or the field interaction with the silicon must be reduced by the use of insulators that separate the metal lines from the silicon [34]. Figure 1 shows a Finite Ground Coplanar (FGC) line fabricated on silicon with a polyimide interface layer, which lifts the transmission line off of the silicon wafer and minimizes field interaction.

The FGC line shown in Figure 1 may be thought of as a Metal-Insulator-Semiconductor (MIS) structure that may support three modes of propagation ( skin effect mode, a dielectric quasi-TEM mode, and a slow-wave mode), and prior work on MIS coplanar waveguides has

George E. Ponchak is with NASA Glenn Research Center, 21000 Brookpark Rd., MS 54/5, Cleveland, OH, 44135. Tel: 216-433-3504; FAX: 216-433-8705; george.ponchak@grc.nasa.gov

John Papapolymerou and Emmanouil M. Tentzeris are with the School of Electrical and Computer Engineering, Georgia Institute of Technology, Atlanta, GA, 30332-0250. explored this with the goal of developing slow wave structures for circuits size reduction [5-7]. However, the slow-wave structures were built on thin insulators deposited over a thin, highly doped semiconductor layer that is grown on an insulating material $[5,6]$. Because the insulating layer is thin (less than $1 \mu \mathrm{m}$ ), the fields interact strongly with the semiconductor layer and the attenuation is reported to be greater than $10 \mathrm{~dB} / \mathrm{cm}[6,7]$. Thus, while interesting and useful for some purposes, the attenuation is too high for most Si RFICs. While references 3 and 4 demonstrated the viability of using thick polyimide interface layers to reduce the dielectric loss of FGC lines and Coplanar Waveguides (CPW) on low resistivity silicon wafers, they did not investigate the influence of the substrate resistivity on the transmission line propagation characteristics. Furthermore, the design rules that were presented do not necessarily yield the lowest attenuation constant.

In this paper, we present for the first time measured propagation constant ( $\alpha$ and $\varepsilon_{\text {eff }}$ ) of FGC lines built on a thick, polyimide interface layer deposited on Si wafers with resistivities that span 0.1 to $15.6 \mathrm{Ohm} \mathrm{cm}$, which covers the range commonly used in CMOS and BiCMOS circuit fabrication. The results are presented to yield design rules for choosing the optimum substrate resistivity and FGC line dimensions.

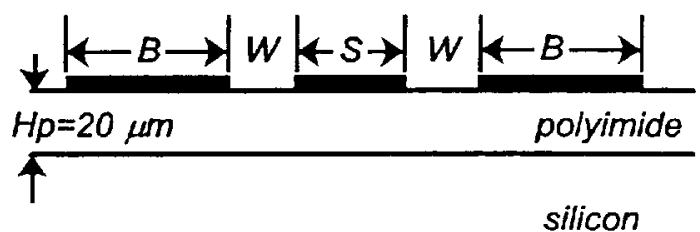

silicon

Figure 1: Cross-sectional cut of Finite Ground Coplanar (FGC) waveguide fabricated on a silicon wafer with a thick, polyimide interface layer.

\section{CIRCUTT FABRICATION AND CHARACTERIZATION}

The resistivity of silicon substrates from three different wafer lots were first measured with a four point probe and determined to be $0.1,1.8$, and $15.60 \mathrm{hm} \mathrm{cm}$. These wafer lots were purposely chosen to encompass the resistivity values commonly used in CMOS and BiCMOS circuits. Dupont adhesion promoter and four, $5 \mu \mathrm{m}$ thick layers of Dupont PI-2611 polyimide were spun onto the

This is a preprint or reprint of a paper intended for presentation at a conference. Because changes may be made before formal publication, this is made available with the understanding that it will not be cited or reproduced without the permission of the author. 
wafer to yield a total polyimide interface layer thickness of $20 \mu \mathrm{m}$; Each layer of polyimide is fully cured at $340 \mathrm{C}$ for 120 minutes before the next layer is added. PI-2611 polyimide has a relative dielectric constant of 3.12 measured at $1 \mathrm{MHz}$ [8] and a loss tangent of 0.002 measured at $1 \mathrm{kHz}$ [9]. The metal FGC circuits consists of $200 \AA \mathrm{Ti}$ and $1.5 \mu \mathrm{m}$ of evaporated $\mathrm{Au}$, both of which are defined by a liftoff process.

The circuits for measuring the propagation constant, $\gamma=\alpha+j k_{0} \sqrt{\varepsilon_{e f f}}$ where $\alpha$ is the attenuation constant and $\varepsilon_{\text {eff }}$ is the effective permittivity, consist of Thru-ReflectLine (TRL) calibration standards with four delay lines of $850,1700,3500$, and $10000 \mu \mathrm{m}$ to cover the frequency band of 1 to $50 \mathrm{GHz}$. The TRL calibration was implemented with MULTICAL [10], TRL software program that computes the propagation constants of the lines by using the difference in the measured magnitude and phase between the thru and delay lines. To improve accuracy, each circuit was measured several times and the average of those measurements are presented in this paper. The measurements were performed on a vector network analyzer and a microwave probe station. While probing, a thick quartz plate was placed between the silicon wafer and the metal wafer chuck, but measurements confirmed that this did not have a measurable influence on the propagation constant.

Sonnet 2-D simulation software was used to choose FGC line dimensions that yield $50 \mathrm{Ohm}$ characteristic impedance on $15 \mathrm{Ohm} \mathrm{cm}$ silicon with the $20 \mu \mathrm{m}$ thick polyimide interface layer. Simulations showed that the characteristic impedance reduced as the resistivity reduced, but within reasonable engineering approximation, the impedance of the lines on all of the substrates may be assumed to be $50 \mathrm{Ohm}$. The dimensions of the FGC lines are shown in Table 1.

Table 1: Dimensions of FGC lines

\begin{tabular}{|l|l|l|l|}
\hline$S(\mu \mathrm{m})$ & $\mathrm{W}(\mu \mathrm{m})$ & $\mathrm{B}=3 \mathrm{~S}(\mu \mathrm{m})$ & $\mathrm{S}+2 \mathrm{~W}(\mu \mathrm{m})$ \\
\hline 51 & 5 & 153 & 61 \\
\hline 62 & 6 & 186 & 74 \\
\hline 74 & 8 & 222 & 90 \\
\hline 90 & 10 & 270 & 110 \\
\hline 114 & 15 & 342 & 144 \\
\hline 120 & 20 & 360 & 160 \\
\hline 140 & 25 & 420 & 190 \\
\hline
\end{tabular}

\section{RESULTS}

The measured attenuation of the FGC lines fabricated on the three silicon wafers are shown in Figure 2. It is seen that the frequency dependence of the attenuation is complicated and highly dependent on the silicon resistivity and FGC line dimensions. When the substrate resistivity is very low $(0.1 \mathrm{Ohm} \mathrm{cm})$, the frequency dependence of attenuation is modeled well by $a=a f^{b}$ where $\mathrm{f}$ is the frequency and $a$ and $b$ are fitting parameters. If conductor loss dominated, $b$ should be 0.5 , but even for the narrowest slot width, $b=0.8$ and increases to 1.4 for the widest slot width, indicating dielectric attenuation is dominating. It is also noted that $b=1$ for the $\mathrm{W}=8 \mu \mathrm{m}(\mathrm{S}+2 \mathrm{~W}=90 \mu \mathrm{m}) \mathrm{FGC}$ line, which agrees with the design rule presented in [4] The $1.8 \mathrm{Ohm} \mathrm{cm}$ silicon substrate has similar frequency dependence, but inflection points are seen that indicate a more complicated frequency dependence. If the substrate resistivity is increased by another order of magnitude to $15.6 \mathrm{Ohm} \mathrm{cm}$, the attenuation appears to be conductor loss dominated, but still indicating the influence of other loss mechanisms. In fact, $\alpha=a f^{b}$ may not be used to model the attenuation.

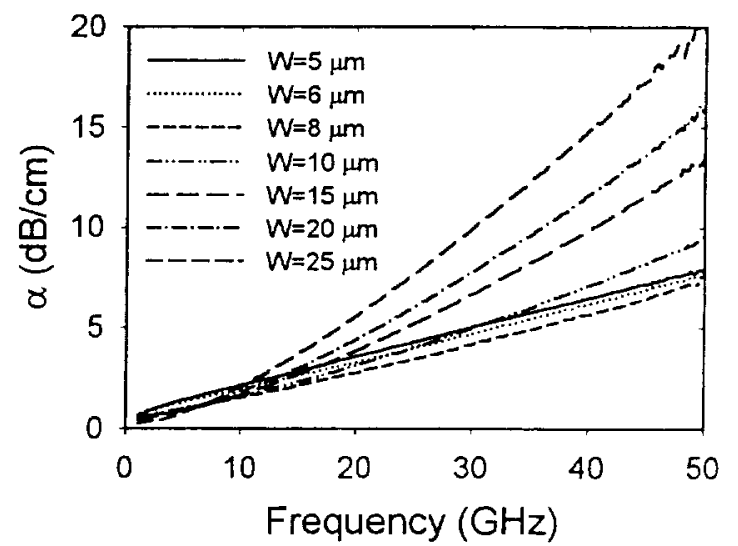

(a) $\rho=0.1 \mathrm{Ohm} \mathrm{cm}$

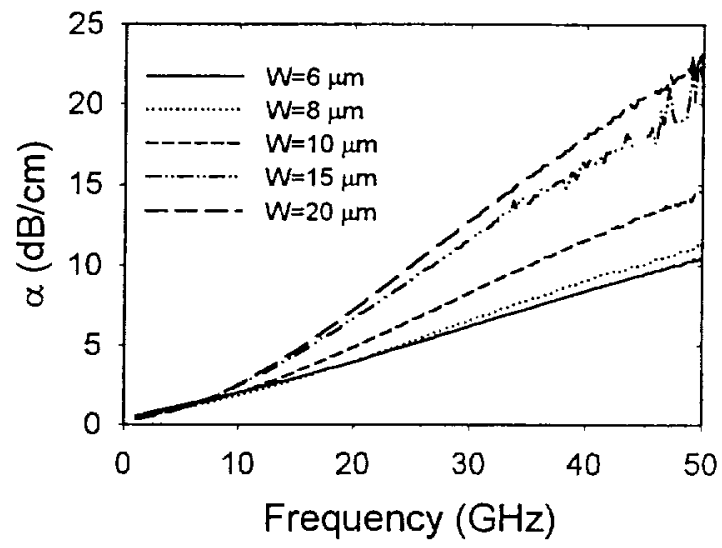

(b) $p=1.8 \mathrm{Ohm} \mathrm{cm}$

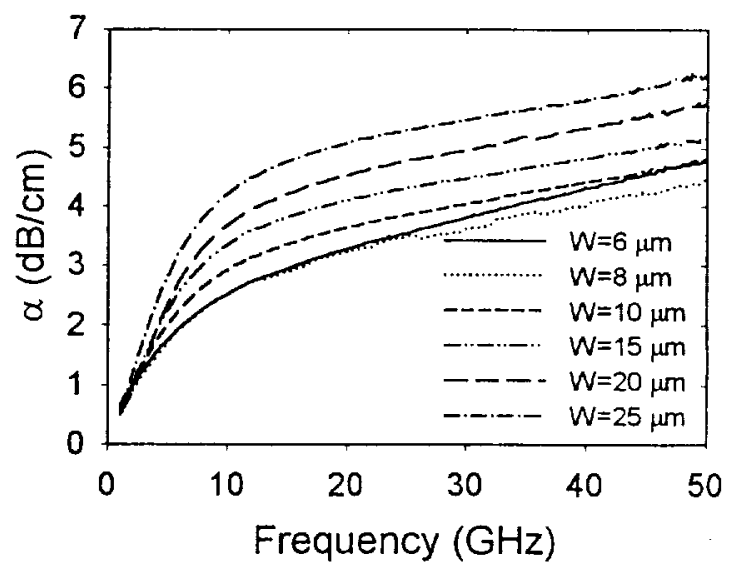

(c) $\rho=15.6 \mathrm{Ohm} \mathrm{cm}$

Figure 2 : Measured attenuation of FGC lines on silicon substrates with resisivities of (a) $0.1 \mathrm{Ohm} \mathrm{cm}$, (b) $1.8 \mathrm{Ohm}$ $\mathrm{cm}$, and (c) $15.6 \mathrm{Ohm} \mathrm{cm}$.

Using the information in Figure 2, the dependence of attenuation on the FGC line geometry and Si resistivity 
may be obtained. This is summarized in Figure 3, which shows the attenuation as a function of $\mathrm{S}+2 \mathrm{~W}$ with the $\mathrm{Si}$ resistivity as a parameter. As seen in Figure 3a, for low frequency (less than several $\mathrm{GHz}$ ), attenuation is more dependent on the strip and slot width than the resistivity, indicating conductor loss dominates. Thus for low frequency circuits, $\mathrm{S}+2 \mathrm{~W}$ should be maximized for low attenuation. For circuits in the microwave region, the FGC dimensions must be chosen to minimize the total loss, which is a combination of the conductor and dielectric loss. From Figures $3 b$ and $3 c$, it is seen that $S+2 W$ approximately equal to $90 \mu \mathrm{m}$ yields the minimum attenuation at both 25 and $40 \mathrm{GHz}$. This is the same value that yielded an approximately linear frequency dependence for low resistivity substrates, and yields a design rule of $(\mathrm{S}+2 \mathrm{~W}) / \mathrm{Hp}=4$ for minimum insertion loss. For smaller line width, conductor loss is higher, but for wider lines, the electric field interaction with the silicon is greater, which increases dielectric loss. Figure 3 also shows the interesting result that $S i$ with a resistivity near 1 Ohm $\mathrm{cm}$ has higher attenuation than resistivities of 0.1 and $10 \mathrm{Ohm} \mathrm{cm}$. A peak in attenuation over a range of substrate resistivity has been predicted for MIS coplanar lines with thin insulator and semiconductor layers [6], but the value of the resistivity for maximum attenuation is different in this case. Lastly, Figure $3 \mathrm{c}$ clearly shows that a substrate resistivity of $10 \mathrm{Ohm} \mathrm{cm}$ or greater is required for acceptable attenuation for a wide range of FGC line widths.

The measured effective permittivity decreased from a high value at $1 \mathrm{GHz}$ to a lower value at $50 \mathrm{GHz}$. The results are summarized in Figure 4 . First, it is seen that at $1 \mathrm{GHz}, \varepsilon_{\text {eff }}$ is approximately the same for each of the three resistivities and increases as the line width increases. In Figure $4 b$, it is seen that $\varepsilon_{\text {eff }}$ also increases with the line width, but at this higher frequency, $\varepsilon_{\text {eff }}$ decreases significantly as the $\mathrm{Si}$ resistivity increases. First, it is expected that $\varepsilon_{\mathrm{eff}}$ should increase as the line width increases because the electric fields are not as tightly bound to the edges of the slot, therefore, they have greater interaction with the Si layer. However, more importantly, the relatively low values of $\varepsilon_{\text {eff }}$ indicate that the thick polyimide layer is effective in minimizing field interaction with the Si.

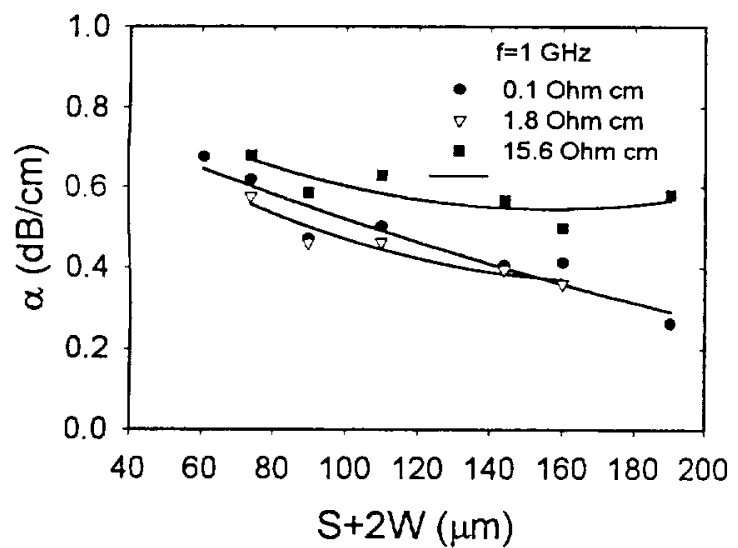

(a)

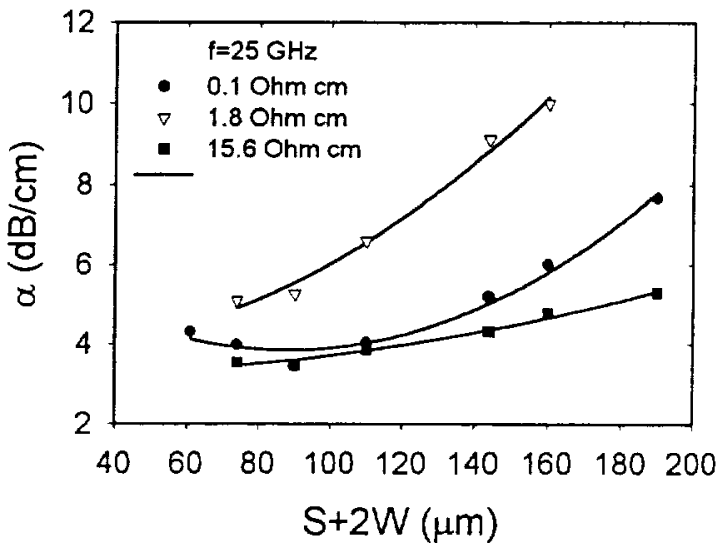

(b)

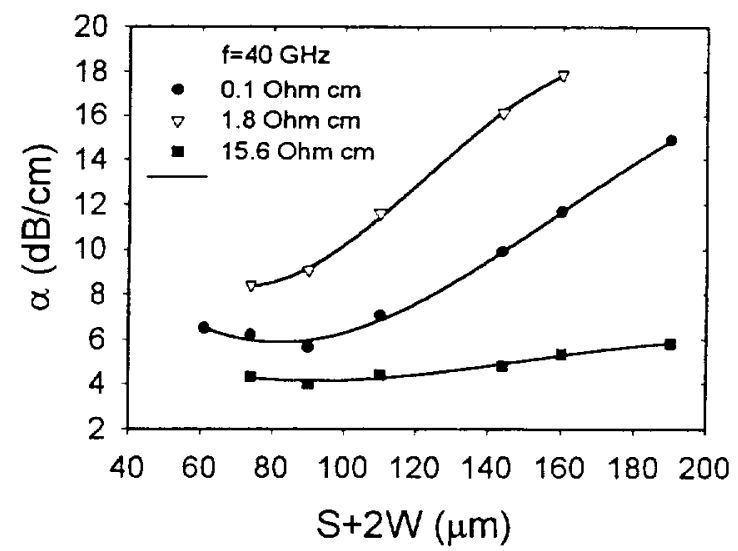

(c)

Figure 3: Measured attenuation as a function of FGC line width and Si resistivity at (a) $1 \mathrm{GHz}$, (b) $25 \mathrm{GHz}$, and (c) 40 GHz.

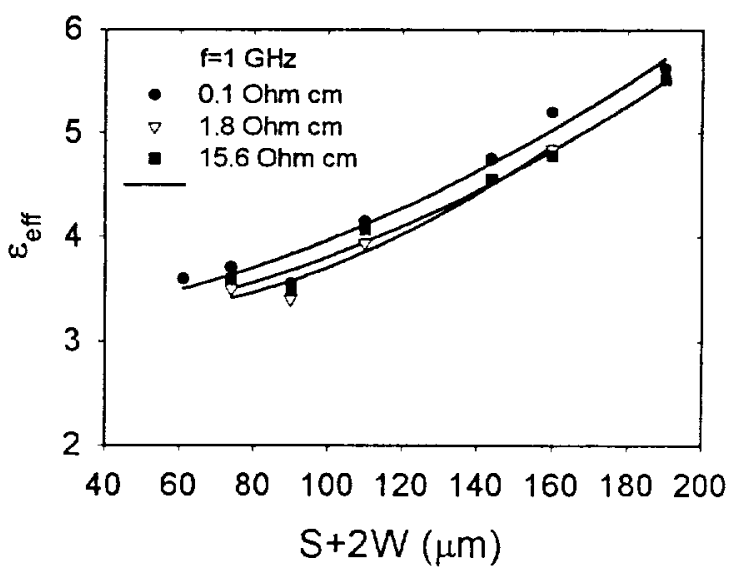

(a) 


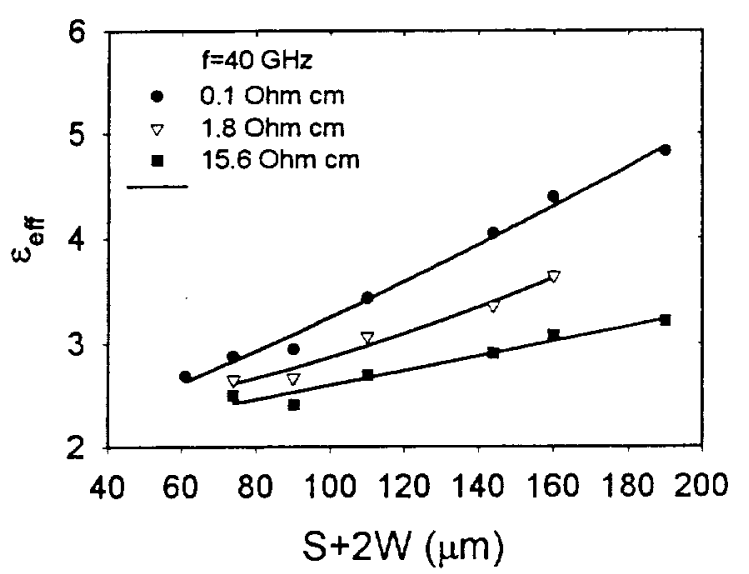

(b)

Figure 4: Measured effective permittivity of FGC lines as a function of the line width with the Si resistivity as a parameter.

[1] G. E. Ponchak, "RF transmission lines on silicon substrates," 29 th European Microwave Conference Dig., Munich, Germany, Oct. 5-7, 1999, pp. 158-161.

[2] G. E. Ponchak and A. N. Downey, "Characterization of thin film microstrip lines on polyimide," IEEE Trans. Components, Packaging, and Manufacturing Tech. -Part B, Vol. 21, No. 2, pp. 171-176, May 1998.

[3] G. E. Ponchak and L. P. B. Katehi, "Measured attenuation of coplanar waveguide on CMOS grade silicon substrates with a polyimide interface layer," IEE Electronics Letters, Vol. 34, No. 13, pp. 1327-1329, June 25, 1998.

[4] G. E. Ponchak, A. Margomenos, and L. P. B. Katehi, "Low loss CPW on low resistivity Si substrates with a micromachined polyimide interface layer for RFIC interconnects," IEEE Trans. Microwave Theory and Techniques, Vol. 49, No. 5, pp. 866-870, May 2001.

[5] R. Sorrentino, G. Leuzzi, and A. Silbermann, "Characteristics of metal-insulator-semiconductor coplanar waveguides for monolithic microwave circuits," IEEE Trans. Microwave Theory and Techniques, Vol. 32, No. 4, pp. 410-416, April 1984.

[6] Yoshiro Fukuoka, Yi-Chi Shih, and Tatsuo Itoh, "Analysis of slow-wave coplanar waveguide for monolithic integrated circuits," IEEE Trans. Microwave Theory and Techniques, Vol. 31, No. 7, pp. 567-573, July 1983.

[7] Tsugumichi Shibata and Eiichi Sano, "Characteristics of MIS structure coplanar transmission lines for investigation of signal propagation in integrated circuits," IEEE Trans. Microwave Theory and Techniques, Vol. 38, No. 7, pp. 881-889, July 1990.

[8] J. Leu, H.-M. Ho, J. K. Lee, J. Kasthurirangan, C. N. Liao, and P. S. Ho, "The evaluation of low dielectric constant materials for deep submicron interconnect applications," in Proc. $6^{\text {th }}$ Meeting Dupont Symp. Polyimide Microelectronics, May 1-3, 1995.

[9] Dupont Company Pyralin LX data sheet.

[10] R. B. Marks, "A multilane method of network analyzer calibration," IEEE Trans. Microwave Theory and Technigues, Vol. 39, pp. 1205-1215, July 1991. 